Ann. Zootech., I965, 14 (3), 247-260.

\title{
SUPPLÉMENTATION PAR LA L-LYSINE D'UN RÉGIME VÉGÉTAL SIMPLIFIÉ CHEZ LE PORG EN CROISSANCE
}

\author{
A. RÉRAT et T. LOUGNON \\ avec la collaboration technique de E. Fngrand, J. M. Borssau et II. Borsquet \\ Station de Recherches sur l'Élevage des Porcs, \\ Centre national de Recherches zootechniques, Jouy-en-Josas (Seine-et-Oise)
}

SOMMAIRE

La supplémentation d'un mélange orge-tourteau d'arachide par le monochlorhydrate de $l$-lysine $(0,2$ p. 10o) dans le régime des porcs de 20 à $60 \mathrm{~kg}$ se traduit par une amélioration significative des critères de croissance (vitesse de croissance, indice de consommation, cocfficient d'efficacité protidique). Les performances des animaux nourris avec ce régime supplémenté égalent celles des porcs consommant un régime isoazoté orge-farine de hareng. La supplémentation à raison de 0,4 p. Ioo de $l$-lysine $\mathrm{HCl}$ du mème régime donne des résultats moins bons sans pourtant qu'aucune différence significative apparaisse entre les performances des animaux des deux lots supplémentés. Au-delà de $60 \mathrm{~kg}$, l'addition de lysine au régime (à raison de 0,3 ou 0,15 p. Ioo) ne permet pas d'obtenir une amélioration significative de la croissance. Les carcasses des animaux supplémentés présentent un pourcentage significativement plus élevé de tissus maigres (jambon + longe) que celles des animaux témoins. Le bilan azoté mesuré sur des porcs mâles castrés confirme les résultats précédents : la rétention azotée s'accroît lorsque le régime orge-arachide est supplémenté par le monochlorhydrate de lysine.

\section{INTRODUCTION}

Une importante fraction des régimes pour les animaux monogastriques en croissance est constituée par des aliments protidiques d'origine animale, tels que la farine de poisson, la farine de viande ou le lait écrémé qui sont fournis dans le but de compléter l'apport azoté des céréales, insuffisant, qualitativement et quantitativement, pour couvrir le besoin azoté. Or, ces aliments présentent généralement une composition en acides aminés suffisamment bonne pour pouvoir être utilisés directement en alimentation humaine et c'est seulement pour certains d'entre eux comme les sous-produits de laiterie ou d'équarissage que devrait être préconisée l'introduction 
dans les rations pour animaux. On est donc amené, en alimentation animale, à substituer, aux protéines animales, des protéines végétales, comme celles des tourteaux oléagineux (arachide, soja, etc.), dont la composition en amino acides essentiels, peu satisfaisante au regard des besoins, doit être améliorée par l'addition de ces substances obtenues par synthèse ou extraction industrielle. C'est ainsi qu'au cours de la dernière décennie, leur production à des prix suffisamment bas pour rendre économiquement possible leur introduction dans les aliments du bétail a suscité de nombreuses recherches concernant la détermination des conditions optimum de supplémentation en fonction des sources azotées utilisées.

Dans cet esprit, compte tenu des conditions du marché français des céréales et des tourteaux, nous avons étudié, au cours d'une série d'expériences, les possibilités d'amélioration de la valeur nutritive d'un mélange de tourteau d'arachide et d'orge par supplémentation à l'aide de $l$-lysine.

\section{MATÉRIEL E'T MÉTHODES}

\section{Animaux et régimes}

Dans une première série d'expériences, les critères étudiés concernaient la croissance, la consommation et la composition corporelle. Cette série a été complétée par la suite par une expérience de bilans au cours de laquelle la digestibilité et la rétention azotée étaient mesurées.

\section{Expériences de croissance.}

Dans chaque expérience, les porcs, de race Large White, pris deux à trois semaines après le sevrage (poids moyen $24 \mathrm{~kg}$ ) sont répartis en blocs formés d'animaux de même sexe, de même poids et issus d'une même portée ; au sein d'un même bloc, chacun des animaux est affecté à un traitement différent. Les régimes sont distribués en alimentation semi ad libilum à raison de trois repas par jour, d'une durée de 20 à 30 minutes chacun. La consommation de nourriture est enregistrée quotidiennement ; les animaux, pesés tous les 15 jours, sont abattus au poids de roo $\mathrm{kg}$. Les carcasses font l'objet de diverses mesures (RÉRAT et HENRY, ig64).

Trois expériences successives ont été effectuées, au cours desquelles les régimes utilisés présentent un taux de I $6 \mathrm{p}$. Ioo et I4 p. Ioo de matières azotées brutes pour les animaux de 20 à $60 \mathrm{~kg}$ et de 60 à $100 \mathrm{~kg}$ respectivement.

Dans la première expérience (expérience A), mettant en jeu 9 blocs de 2 animaux, le tourteau d'arachide est comparé à la farine de hareng de Norvège en tant que supplément azoté d'un régime à base de céréales.

Dans une autre expérience (expérience $\mathrm{C}$ ), mettant en jeu 9 blocs de 3 animaux, les effets de l'introduction de $l$-lysine à deux niveaux dans le régime précédent (orge-arachide) sont étudiés: l'un des taux utilisés rééquilibre le déficit théorique vis-à-vis des besoins (BECKER, I958), l'autre représente un excès d'acide aminé. Un apport variable d'un acide aminé non indispensable, le glycocolle, est effectué pour maintenir constant le taux d'azote aminé libre dans le régime.

C'est pourquoi, dans une autre expérience (expérience B), mettant en jeu 5 blocs de 2 animaux, est étudiée l'influence de l'incorporation d'un acide aminé non indispensable, le glycocolle, sur la valeur nutritive d'un régime orge-arachide.

La composition des régimes utilisés au cours de ces trois expériences est rapportée dans les tableaux I et 2.

\section{Expérience de bilans.}

Dans cette expérience (expérience D), qui a pour objet de compléter l'expérience C, 4 blocs de trois animaux mâles de la même portée et de même poids ont été utilisés. Les animaux sont placés dans des cages individuelles munies d'un dispositif permettant la collecte séparée des fèces et de l'urine. Contrairement aux expériences de croissance, l'alimentation est rationnée en fonction du poids vif des animaux et de telle manière que l'ingestion soit identique pour les 3 animaux du 


\section{TABLEAU I}

Composition des régimes utilisés dans les expériences $A, B, C$ et $D$ (Animaux de 20 à $60 \mathrm{~kg}$ )

\begin{tabular}{|c|c|c|c|c|c|c|c|}
\hline \multirow[b]{2}{*}{ Lots $\ldots \ldots \ldots \ldots \ldots \ldots \ldots$} & \multicolumn{2}{|c|}{ Expérience A } & \multicolumn{2}{|c|}{ Expérience B } & \multicolumn{3}{|c|}{ Expériences $\mathrm{C}$ et $\mathrm{D}$} \\
\hline & I & II & I & II & $\mathrm{I}$ & II & III \\
\hline Orge $\ldots \ldots \ldots \ldots \ldots \ldots \ldots \ldots$ & 80,0 & 80,0 & 80,0 & 83,0 & 80,0 & 80,0 & 80,0 \\
\hline Tourteau d'arachide ........... & - & 12,4 & 12,4 & $11 t, 0$ & 12,4 & 12,4 & 12,4 \\
\hline Farine de poisson ............ & 9,2 & - & - & - & - & - & - \\
\hline 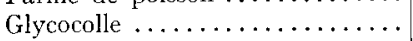 & 1,0 & 1,0 & 1,0 & - & 1,0 & 0,9 & 0,7 \\
\hline L-lysine monochlorhydrate ..... & - & - & - & - &.- & $0,2^{*}$ & $0,4^{* * *}$ \\
\hline Amidon..$\ldots \ldots \ldots \ldots \ldots$ & 6,8 & 3,6 & 3,6 & - & 3,6 & 3,5 & 3,5 \\
\hline Mélange minéral et vitaminique ${ }^{* * *}$ & 3,0 & 3,0 & 3,0 & 3,0 & 3,0 & 3,0 & 3,0 \\
\hline \multicolumn{8}{|l|}{ Composition chimique p. 100} \\
\hline Matières azotées brutes $(\mathrm{N} \times 6,25)$ & \multicolumn{2}{|c|}{16,1} & \multicolumn{2}{|c|}{16,0} & \multicolumn{3}{|c|}{17,3} \\
\hline Matières cellulosiques .......... & \multicolumn{2}{|c|}{3,5} & \multicolumn{2}{|c|}{4,5} & \multicolumn{3}{|c|}{4,5} \\
\hline Matières grasses ............... & \multicolumn{2}{|c|}{2,9} & \multicolumn{2}{|c|}{2,1} & \multicolumn{3}{|c|}{2,0} \\
\hline 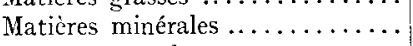 & \multirow{2}{*}{\multicolumn{2}{|c|}{4,4}} & \multicolumn{2}{|c|}{3,7} & \multicolumn{3}{|c|}{4,3} \\
\hline UF pour $100 \mathrm{~kg} \ldots \ldots \ldots \ldots \ldots$ & & & & & & 100 & \\
\hline
\end{tabular}

* Soit 0,13 p. 100 de lysine pure.

** Soit 0,27 p. 100 de lysine pure.

*** Composition rapportée par ailleurs (RÉRAT, Lougnon et PIon, 1962).

\section{TABIEAU 2}

Composition des régimes utilisés dans les expériences $A, B, C$ et $D$

(Animaux de 60 à $100 \mathrm{~kg}$ )

\begin{tabular}{|c|c|c|c|c|c|c|c|}
\hline \multirow[b]{2}{*}{ Lots $\ldots \ldots \ldots \ldots \ldots \ldots \ldots \ldots$} & \multicolumn{2}{|c|}{ Expérience A } & \multicolumn{2}{|c|}{ Expérience B } & \multicolumn{3}{|c|}{ Expériences $\mathrm{C}$ et $\mathrm{D}$} \\
\hline & I & II & I & II & I & II & III \\
\hline Orge $\ldots \ldots \ldots \ldots \ldots \ldots \ldots \ldots$ & 80,0 & 80,0 & 80,0 & 83,0 & 80,0 & 80,0 & 80,0 \\
\hline Tourteau d'arachide ........... & 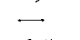 & 8,8 & 8,8 & 10,0 & 8,8 & 8,8 & 8,8 \\
\hline Farine de poisson $\ldots \ldots \ldots \ldots \ldots$ & 6,5 & - & - & - & 一 & - & 一 \\
\hline 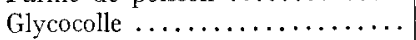 & 1,0 & 1,0 & 1,0 & - & 1,0 & 0,95 & 0,8 \\
\hline L-Iysine monochlorhydrate ..... & 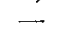 & - & - & - & - & $0,15^{*}$ & $0,3^{* *}$ \\
\hline Amidon $\ldots \ldots \ldots \ldots \ldots \ldots \ldots$ & 9,5 & 7,2 & 7,2 & 4,0 & 7,2 & 7,1 & 7,1 \\
\hline Mélange minéral et vitaminique $\mathrm{e}^{* *}$ & 3,0 & 3,0 & 3,0 & 3,0 & 3,0 & 3,0 & 3,0 \\
\hline \multicolumn{8}{|l|}{ Composition chimique p. 100} \\
\hline Matières azotées brutes $(\mathrm{N} \times 6,25)$ & \multicolumn{2}{|c|}{14,3} & \multicolumn{2}{|c|}{14,4} & \multicolumn{3}{|c|}{14,2} \\
\hline Matières cellulosiques .......... & \multicolumn{2}{|c|}{4,0} & \multicolumn{2}{|c|}{3,9} & \multicolumn{3}{|c|}{4,5} \\
\hline Matières grasses ............. & \multicolumn{2}{|c|}{3,0} & \multicolumn{2}{|c|}{2,2} & \multicolumn{3}{|c|}{2,0} \\
\hline Matières minérales .............. & \multicolumn{2}{|c|}{5,2} & \multicolumn{2}{|c|}{4,1} & \multicolumn{3}{|c|}{4,0} \\
\hline UF pour $100 \mathrm{~kg} \ldots \ldots \ldots \ldots$ & \multicolumn{2}{|c|}{101} & \multicolumn{2}{|c|}{103} & \multicolumn{3}{|c|}{100} \\
\hline
\end{tabular}

* Soit 0,10 p. 100 de lysine pure.

** Soit 0,20 p. 100 de lysine pure.

*** Composition rapportée par ailleurs (RÉRAT, Lougnon et PIon, 1962). 
même bloc (méthode paired-feeding de MircirelL, I930). Les porcs sont pesés une fois par semaine.

La récolte des fèces et de l'urine est faite par périodes de 6 jours, séparées par une journée d'interruption. Les fèces sont récoltées chaque jour, pesées et séchées dans une étuve à ventilation à $70^{\circ} \mathrm{C}$. Le dosage de l'azote (Kjeldahl) est effectué sur un échantillon représcntatif de l'ensemble des récoltes de la semaine. Les urines sont recueillies dans une solution d'acide sulfurique $6 \mathrm{~N}$. Chaque jour, une partie aliquote $(\mathrm{I} / 20 \mathrm{ou} \mathrm{I/30})$ de l'émission est prélevée et stockée à $4^{\circ} \mathrm{C}$. Le dosage de l'azote est effectué sur la somme des 6 prélèvements préalablement ajustée à un volume connu (I litre).

\section{Méthodes analytiques}

- Les techniques utilisées pour analyser les régimes (azote, matières grasses, matière sèche, cendres) ont déjà été décrites par ailleurs (RÉrat, Lougnon et PION, ig6z).

- Les amino acides des constituants azotés des régimes (orge, tourteau d'arachide, farine de poisson), ont été dosés (PIon, I963) par chromatographie sur colonne selon la méthode indiquée précédemment (RÉRAT, IJOUGNON et PION, I962).

- Le contrôle des doses de lysine additionnelle a été réalisé par extraction par l'eau et dosage microbiologique : méthode de DunN et al. (I944), modifiée par Fauconneat (communication personnelle). 1958).

- Enfin, les calculs statistiques ont été réalisés selon la méthode d'analyse de variance (Lison,

\section{RÉSULTATS}

\section{Composition chimique des régimes}

Les principaux résultats đes analyses des régimes figurent dans les tableaux I et 2 . Le tableau 3 donne les teneurs en amino acides des trois sources protidiques utilisées (orge, tourteau d'arachide, farine de poisson).

Compte-tenu de ces données, la composition en acides aminés indispensables du régime orge-arachide a été calculée et comparée à celle du régime orge-poisson (tabl. 4 et 5 ) aux deux niveaux azotés.

\section{TABLEAU 3}

Composition en amino acides des constituants arotés des régimes (en g pour $16 \mathrm{~g}$ d'azote) (PION et al., 1963)

\begin{tabular}{|c|c|c|c|}
\hline & Orge & $\begin{array}{l}\text { Tourteau } \\
\text { d'arachide }\end{array}$ & $\begin{array}{c}\text { Farine } \\
\text { de hareng } \\
\text { de Norvège }\end{array}$ \\
\hline Arginine $\ldots \ldots \ldots \ldots \ldots \ldots \ldots$ & 4,8 & $11, t^{\prime}$ & 4,9 \\
\hline Ilistidine $\ldots \ldots \ldots \ldots \ldots \ldots \ldots$ & 2,2 & 2,2 & 2,2 \\
\hline Isoleucine $\ldots \ldots \ldots \ldots \ldots \ldots$ & 3,7 & $3, \mathbf{y}^{\prime}$ & $1,{ }^{\prime}$ \\
\hline Leucine . . . . . . . . . . . & 6,8 & 6,05 & 7,1 \\
\hline Lysine $\ldots \ldots \ldots \ldots \ldots \ldots \ldots$ & 3,7 & 3,4 & 7,25 \\
\hline Méthionine $\ldots \ldots \ldots \ldots \ldots \ldots$ & 1,4 & 0,7 & 2,55 \\
\hline Cystine..$\ldots \ldots \ldots \ldots \ldots \ldots \ldots$ & 2,7 & 1,5 & 0,9 \\
\hline Méthionine + cystine ........ & 4,1 & 2,2 & 3,45 \\
\hline Phénylalanine ............ & 4,6 & 4,5 & 3,7 \\
\hline 'Tyrosine .................. & 3,1 & 3,8 & 9,75 \\
\hline 'lhréonine . . . . . . . & 3,4 & 2,6 & 4,3 \\
\hline Tryptophane ........ & 1,0 & 0,9 & 1,0 \\
\hline 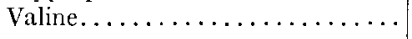 & 5,5 & 4,4 & 5,75 \\
\hline
\end{tabular}




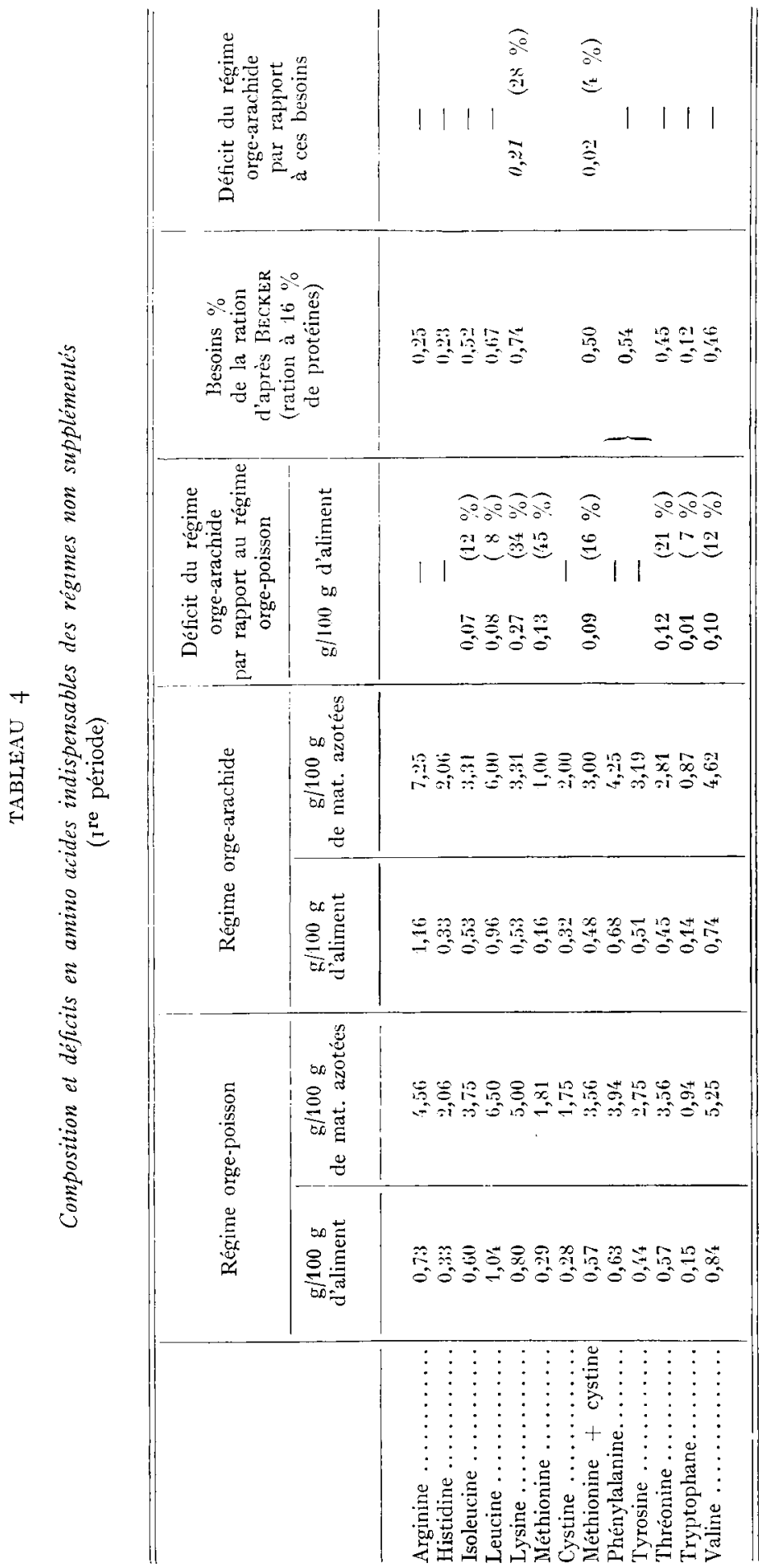




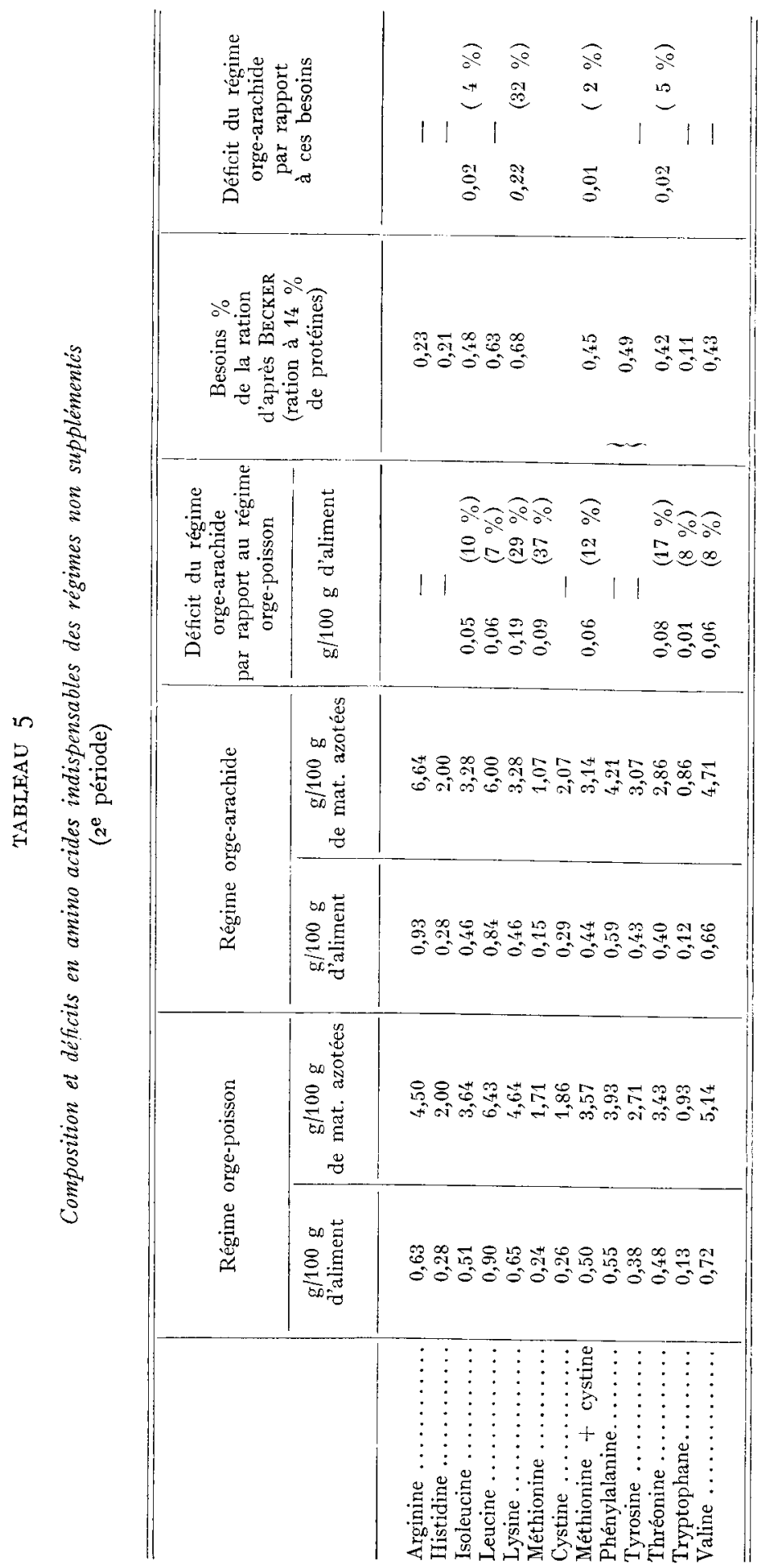


Par rapport au régime orge-poisson, le régime orge-arachide est essentiellement déficient en lysine et secondairement en thréonine. Par rapport aux besoins en acides aminés définis par BEckER (I958), seul subsiste un déficit marqué en lysine et ceci aussi bien au cours de la première période de croissance que pendant la deuxième période ; les doses respectives de $2 \mathrm{~g}$ et $\mathrm{I}, 5 \mathrm{~g}$ de $l$-lysine par $\mathrm{kg}$ de régime comblent théoriquement ce déficit au cours de chacune de ces périodes.

\section{Expériences de croissance}

A. Croissance et consommation (tabl. 6).

Le remplacement, à taux isoazoté, de la farine de poisson par du tourteau d'arachide (expérience A) provoque une diminution de la valeur nutritive des régimes

TABLEAU 6

Croissance et consommation. Résultats moyens

\begin{tabular}{|c|c|c|c|c|c|c|c|}
\hline \multirow[b]{2}{*}{ Lot $\ldots \ldots \ldots \ldots \ldots \ldots \ldots \ldots$} & \multicolumn{2}{|c|}{ Expérience A } & \multicolumn{2}{|c|}{ Expérience $B$} & \multicolumn{3}{|c|}{ Expérience (; } \\
\hline & $\mathrm{I}_{\Lambda}$ & $\mathrm{II}_{\mathrm{A}}$ & $I_{B}$ & $\mathrm{II}_{\mathrm{B}}$ & $\mathbf{I}_{\mathrm{C}}$ & $\mathrm{II}_{\mathrm{G}}$ & $\operatorname{III}_{\mathrm{C}}$ \\
\hline Régime de base : orge $+\ldots \ldots$ & Poisson & Arachide & Arachide & Arachide & Arachide & Arachide & Arachide \\
\hline $\begin{array}{l}\text { Glycocolle supplémentaire .. } \\
\text { L-lysine supplémentaire ... }\end{array}$ & + & + & $\stackrel{+}{-}$ & - & $\stackrel{+}{+}$ & + & $\frac{+}{+}$ \\
\hline $\begin{array}{l}\text { Période de } 25-60 \mathrm{~kg} \\
\text { (taux azoté } 16 \%)\end{array}$ & & & & & & & \\
\hline Gain moyen quotidien $(g)$ & 503 & $358^{*}$ & $45^{\prime} t$ & 439 & 365 & 496 & 452 \\
\hline Consommation journalière $(\mathrm{kg})$ & 1,61 & $1,49^{*}$ & 1,68 & 1,67 & 1,42 & 1,60 & 1.49 \\
\hline Indice de consommation (1) ... & 3,22 & $4,17^{*}$ & 3,70 & $\overrightarrow{3,80}$ & 3,90 & 3,26 & 3,31 \\
\hline Coefficient d'efficacité proticlique ( ${ }^{(2)}$ & 1,95 & $1,50^{*}$ & 1,69 & $1,1,4$ & 1,60 & 1,93 & 1,89 \\
\hline $\begin{array}{l}\text { Période de } 60-100 \mathrm{~kg} \\
\text { (taux azoté } \mathbf{1 4} \% \text { ) }\end{array}$ & & & & & & & \\
\hline Gain moyen quotidien (g) & 675 & 752 & $60_{4}^{\prime}$ & 630 & 735 & 738 & $75 \overline{1}$ \\
\hline Consommation journalière $(\mathrm{kg}) \ldots$ & $\overline{2,49}$ & $2,91^{*}$ & $2,5.3$ & 2,47 & $\overline{2,78}$ & 2,88 & 2,63 \\
\hline Indice de consommation $\left({ }^{(}\right) \ldots$ & 3,73 & 3,89 & 4,21 & 3,96 & 3,79 & 3,91 & 3,54 \\
\hline Coefficient d'efficacité protidlique ( $\left.{ }^{2}\right)$ & 1,92 & 1,81 & 1,70 & 1,81 & 1,88 & 1,83 & 2,04 \\
\hline Période totale $25-100 \mathrm{~kg}$ & & & & & & & \\
\hline Gain moyen journalier $(g) \ldots$ & 573 & $487^{*}$ & 517 & 517 & $19 \%$ & 591 & 566 \\
\hline Consommation journalière $(\mathrm{kg}) \ldots$ & 1,99 & 1,96 & $2,0^{\prime}$ & 2,01 & 1,89 & 2,11 & 1,94 \\
\hline Indice de consommation $(1) \ldots$ & 3,49 & $4,03^{*}$ & 3,96 & $3, \overline{3,89}$ & $3 \overline{3,85}$ & 3,57 & $3,4,4$ \\
\hline Coefficient d'efficacité protidique $\left.{ }^{(2}\right)$ & 1,93 & $1,65^{*}$ & 1,69 & 1,72 & $1,7 / t$ & 1,88 & $1, \overline{96}$ \\
\hline
\end{tabular}

$\left(^{*}\right)$ Différences significatives au seuil de $1 \%$.

(1) Indice de consommation : $\frac{\text { quantité d'aliment ingérée }(\mathrm{kg})}{\text { gain de poids }(\mathrm{kg})}$.

$\left({ }^{2}\right)$ Coefficient d'efficacité protidique : $-\frac{\text { gain de poids }}{(\mathrm{kg} g)} \frac{-}{\text { quantité de protéines ingérée }(\mathrm{kg})}$.

Les différences non significatives au seuil de $5 \%$ sont reliées par un trait. 
à base d'orge : la vitesse de croissance des animaux est diminuée, l'indice de consommation et le coefficient d'efficacité protidique deviennent mauvais. Les différences enregistrées entre les deux lots d'animaux pour ces différents critères sont significatives pour l'ensemble de la croissance; mais c'est essentiellement parce qu'elles sont très marquées durant la première phase de croissance. Par contre, durant la deuxième phase les animaux recevant de l'arachide ont tendance à croître plus vite que ceux recevant du poisson. L'inversion des performances des animaux lors des deux phases de croissance peut être attribuée à la consommation journalière : elle est la plus élevée lors de la première période pour les animaux recevant du poisson, et lors de la deuxième période pour ceux recevant de l'arachide comme complément azoté.

L'addition de glycocolle, acide aminé non essentiel, au régime orge-arachıde, ne modifie en aucune façon le niveau d'alimentation et la vitesse de croissance des animatux (expérience $B$ ).

L'addition de lysine améliore la valeur alimentaire du régime orge-arachide (expérience C) pendant les premières phases de la croissance. Cette action, tant sur la vitesse de croissance que sur l'indice de consommation, disparaît lorsque les animaux atteignent $60 \mathrm{~kg}$ de poids vif. C'est à raison de $2 \mathrm{~g}$ par $\mathrm{kg}$ de ration que la lysine provoque les meilleures performances des animaux; la dose de $4 \mathrm{~g}$ semble, au moins au début de la croissance, être moins efficace tout en ayant une action bénéfique par rapport au régime témoin.

D'après la comparaison des expériences $\mathrm{A}$ et $\mathrm{C}$, dans lesquelles les animaux recevant le même régime $\left(\mathrm{II}_{\mathrm{A}}\right.$ et $\mathrm{I}_{\mathrm{C}}$ ) présentent le même comportement (mêmes moyennes), le tourteau d'arachide additionné de $0,2 \mathrm{p}$. Ioo de lysine est aussi efficace que la farine de poisson en tant que supplément azoté des régimes pour Porc.

\section{B. Résultats d'abattage (tab1. 7).}

La variabilité de la composition corporelle est très élevée si bien qu'il n'existe pas de différence significative entre les lots dans chaque expérience; une exception toutefois concerne le pourcentage des morceaux maigres qui s'élève lorsque le régime orge-arachide est additionné de lysine (expérience $\mathrm{C}$ ). Cette tendance à l'amaigrissement est retrouvée au niveau des autres critères chez les mêmes animaux. Par ailleurs, il apparaît également que dans l'expérience $A$, les animaux recevant de l'orge supplémentée de poisson sont moins gras que ceux recevant des régimes orgearachide.

\section{Expérience de bilans}

Période de 30 à $60 \mathrm{~kg}$ (tabl. 8).

Les animaux recevant les régimes supplémentés par la $l$-lysine présentent, visà-vis des témoins, une plus grande vitesse de croissance et une rétention azotée sensiblement améliorée en valeur absolue et en valeur relative ; leur consommation journalière est la même que celle des témoins, ainsi que cela était prévu par le protocole expérimental. Ni la digestibilité de la matière sèche, ni la digestibilité azotée ne sont modifiées par la présence de lysine dans le régime; à noter toutefois que l'ingestion azotée journalière est, dans ce cas, légèrement augmentée. Enfin, l'effet "bloc " est statistiquement très marqué pour la plupart des critères au cours de cette 
TABLEAU 7

Composition corporelle. Résultats moyens

\begin{tabular}{|c|c|c|c|c|c|c|c|}
\hline \multirow[b]{2}{*}{ Lot $\ldots \ldots \ldots \ldots \ldots \ldots \ldots$} & \multicolumn{2}{|c|}{ Expérience $\mathrm{A}$} & \multicolumn{2}{|c|}{ Expérience 13} & \multicolumn{3}{|c|}{ Expérience C } \\
\hline & $\mathrm{I}_{\mathrm{A}}$ & $\mathrm{II}_{\mathrm{A}}$ & $\mathrm{I}_{\mathbf{B}}$ & $\mathrm{II}_{13}$ & $\mathrm{I}_{\mathrm{C}}$ & $\mathrm{II}_{\mathrm{C}}$ & $\mathrm{III}_{\mathrm{C}}$ \\
\hline $\begin{array}{l}\text { Nature du supplément azoté } \ldots \ldots \\
\text { Glycocolle } \ldots \ldots \ldots \ldots \ldots \ldots \ldots \ldots \\
\text { L-lysine } \ldots \ldots \ldots \ldots \ldots \ldots \ldots\end{array}$ & $\begin{array}{l}\text { Poisson } \\
+ \\
+\end{array}$ & $\begin{array}{l}\text { Arachide } \\
+ \\
+\end{array}$ & $\begin{array}{c}\text { Arachide } \\
+ \\
-\end{array}$ & $\begin{array}{l}\text { Arachide } \\
- \\
-\end{array}$ & $\begin{array}{l}\text { Arachide } \\
+ \\
+\end{array}$ & $\begin{array}{c}\text { Arachide } \\
+ \\
0,2\end{array}$ & $\begin{array}{l}\text { Arachide } \\
+ \\
0,1\end{array}$ \\
\hline Rendement $(\%) \ldots \ldots \ldots \ldots \ldots$ & 72,8 & 71,8 & $\underline{72,9}$ & 73,9 & 73,0 & 71,7 & 71,9 \\
\hline$\frac{\text { Jambon }+ \text { longe }}{\text { Poids net }} \times 100 \ldots \ldots \ldots$ & 51,5 & 49,9 & 48,4 & 49,2 & 48,4 & 49,2 & 50,7 \\
\hline$\frac{\text { Bardière }+ \text { panne }}{\text { Poids net }} \times 100 \ldots \ldots$ & 18,6 & 20,1 & 21,8 & $21, \mathbf{k}$ & $\underline{21,9}$ & 21,6 & 20,2 \\
\hline Épaisseur du lard (mm) (1) .... & 28,7 & 30,3 & 33,0 & 29,0 & 38,0 & 32,1 & 29,1 \\
\hline
\end{tabular}

Les moyennes reliées par un trait ne présentent pas de différence significative.

(1) Épaisseur du lard : moyenne de l'épaisseur au dos et de l'épaisseur au rein.

TABLEAU 8

Digestibilité et bilan (Expérience D). Résultats moyens (4 animaux par lot) Animaux entre 30 et $60 \mathrm{~kg}$ de poids vif

\begin{tabular}{|c|c|c|c|c|}
\hline Lot $\ldots \ldots \ldots \ldots \ldots \ldots \ldots \ldots \ldots \ldots$ & I & II & III & \\
\hline Régime $\ldots \ldots \ldots \ldots \ldots \ldots \ldots \ldots \ldots \ldots \ldots$ & $\begin{array}{c}\text { Régime de base } \\
\text { (R. B.) }\end{array}$ & $\begin{array}{c}\text { R. B. } \\
+0,2 \% \\
\text { l-lysine }\end{array}$ & $\begin{array}{c}\text { R. B. } \\
+\quad 0,4 \% \\
\text { l-lysine }\end{array}$ & $\begin{array}{l}\text { Signification } \\
\text { statistique (1) }\end{array}$ \\
\hline $\begin{array}{l}\text { Gain moyen quotidien }(g) \ldots \ldots \ldots \ldots \\
\text { Consommation quotidienne }(\mathrm{kg}) \ldots \ldots \ldots \\
\text { Matière sèche fécale quotidienne }(\mathrm{g}) \ldots \ldots \\
\text { C.U.D. matière sèche } \mathrm{p} .100 \ldots \ldots \ldots \ldots \ldots \\
\text { Azote ingéré par jour }(\mathrm{g}) \ldots \ldots \ldots \ldots \ldots \\
\text { Azote fécal quotidien }(\mathrm{g}) \ldots \ldots \ldots \ldots \ldots \ldots \\
\text { C.U.D. azoté p. } 100 \ldots \ldots \ldots \ldots \ldots \ldots \\
\text { Azote urinaire journalier }(\mathrm{g}) \ldots \ldots \ldots \ldots \\
\text { Azote retenu par jour }(\mathrm{g}) \ldots \ldots \ldots \ldots \ldots \\
\frac{\mathrm{N} \text { retenu }}{\mathrm{N} \text { absorbé }} \text { p. } 100 \ldots \ldots \ldots \ldots \ldots \ldots\end{array}$ & $\begin{array}{l}420 \\
1,45 \\
235 \\
83,7 \\
41,7 \\
6,95 \\
83,3 \\
20,5 \\
14,2 \\
41,0\end{array}$ & $\begin{array}{c}560 \\
1,58 \\
259 \\
83,6 \\
45,3 \\
8,13 \\
82,1 \\
20,3 \\
17,1 \\
44,7\end{array}$ & $\begin{array}{l}570 \\
1,55 \\
215 \\
84,0 \\
44,5 \\
7,85 \\
82,3 \\
17,8 \\
18,9 \\
50,9\end{array}$ & $\begin{array}{l}L^{* *} B^{*} \\
B^{* *} \\
\text { N.S. } \\
\text { N.S. } \\
B^{*} \\
\text { L* B* } \\
\text { N.S. } \\
\text { N.S. } \\
\text { L* B* } \\
\text { L* B* }\end{array}$ \\
\hline
\end{tabular}

(1) Analyse statistique :

$\mathrm{L}$ : effet linéaire du traitement significatif à $5 \%\left(^{*}\right)$ ou à $1 \%$ (*) $^{*}$.

$\mathrm{B}$ : effet bloc significatif à $5 \%\left(^{*}\right)$ ou d̀ $1 \%\left(^{* *}\right)$.

N.S. : effet non significatif. 
période, et ce fait peut être attribué à la méthode d'égalisation de la consommation utilisée pour l'estimation des bilans.

Période de 60 à go $\mathrm{kg}$ (tabl. 9).

Au cours de cette période, par contre, l'effet bloc disparaît. En outre, compte tenu de la faible consommation du lot témoin, il n'a pas été possible d'égaliser complètement les niveaux d'ingestion et la consommation de matière sèche et d'azote des animaux recevant les régimes supplémentés en lysine est sensiblement supérieure à celle des animaux recevant le régime de base; le gain moyen journalier de ces animaux est plus élevé que celui des témoins ; il en est de même de la rétention absolue d'azote. Par ailleurs, l'addition de lysine au régime de base se traduit par un meilleur rendement de l'azote absorbé (environ I5 p. Ioo), cependant que la digestibilité azotée n'est pas modifiée.

TABLEAU 9

Digestibilité et bilan (Expérience D). Résultats moyens (4 animaux par lot) Animaux entre 60 et $90 \mathrm{~kg}$

\begin{tabular}{|c|c|c|c|c|}
\hline I.ot $\ldots \ldots \ldots \ldots \ldots \ldots \ldots \ldots \ldots \ldots$ & I & II & III & \\
\hline Régine $\ldots \ldots \ldots \ldots$ & $\begin{array}{l}\text { Régime de base } \\
\text { (R. 13.) }\end{array}$ & $\begin{array}{c}\text { R. B. } \\
+0,15 \% \\
\text { l-lysine }\end{array}$ & $\begin{array}{c}\text { R. B. } \\
+0,3 \% \\
\text { l-lysine }\end{array}$ & $\begin{array}{l}\text { Signification } \\
\text { statistique }\left(^{1}\right)\end{array}$ \\
\hline Gain moyen quotidien $(\mathrm{g})$ & 559 & 763 & 738 & $\mathrm{~L}^{* *} \mathrm{~B} *$ \\
\hline Consommation quotidienne (kg) & 2,12 & 2,47 & 2,32 & $L^{* *}$ \\
\hline Matière sèche fécale joumalière (g) .. & 313 & 368 & 351 & $\mathrm{~L}^{*}$ \\
\hline C.U.D. matière sèche p. $100 \ldots \ldots \ldots$. & 85,3 & 85,1 & 84,8 & N.S. \\
\hline Azote ingéré par jour $(\mathrm{s}) \ldots \ldots \ldots \ldots$ & 54,4 & 63,2 & $59, l_{4}$ & $\mathrm{~L}^{* *}$ \\
\hline Azote fécal quotidien $(\mathrm{g}) \ldots \ldots \ldots \ldots$ & 8,2 & 10,4 & 9,3 & N.S. \\
\hline C.U.D. azote p. $100 \ldots \ldots \ldots \ldots \ldots \ldots$ & 8,5 & 83,6 & $8{ }^{\prime}, 0$ & N.S. \\
\hline Azote urinaire journalier $(\mathrm{g}) \ldots \ldots \ldots \ldots$ & 28,6 & 29,1 & 27,8 & N.S. \\
\hline Azote retenu par jour $(g) \ldots \ldots \ldots$ & 17,5 & 23,7 & 22,0 & $\mathrm{~L}^{*}$ \\
\hline$\frac{N \text { retenu }}{\mathrm{N} \text { absorbé }}, 100 \ldots \ldots \ldots \ldots$ & 38,0 & 44,8 & 44,0 & $\mathrm{~L}^{*}$ \\
\hline
\end{tabular}

(1) Analyse statistique : (voir tableau 8)

\section{DISCUSSION}

Il est bien connu qu'en raison de leur médiocre teneur en azote et de leur composition déséquilibrée en amino acides essentiels, les céréales incorporées aux régimes pour animaux réclament un apport complémentaire pour couvrir le besoin azoté, tant sur le plan quantitatif que sur le plan qualitatif. A cet égard, ce sont les protéines d'origine animale, riches en lysine, telles que la farine de poisson ou le lait écrémé, 
qui conviennent le mieux puisqu'elles viennent combler le déficit primaire en cet acide aminé dont le pourcentage vis-à-vis des protéines d'œuf (BL,Ock et MITCHELL, I946) atteint $48 \mathrm{p}$. Ioo dans le cas de l'orge. On peut présumer, par contre, que le tourteau d'arachide ne peut rééquilibrer convenablement les céréales puisqu'il présente deux déficits importants (lysine : 52 p. Ioo, acides aminés soufrés : 67 p. roo). De fait, nos propres résultats, montrant que l'orge est moins efficacement supplémentée par le tourteau d'arachide que par la farine de poisson, viennent confirmer cette hypothèse déduite de la composition chimique de ces protéines. Ils sont en accord avec ceux d'Evans (I958, I959, I960, Ig6I) et de JonEs et al. (I96I, I962) De la même façon, le tourteau d'arachide est inférieur à celui du soja comme complément azoté d'un régime à base d'orge (Clausen et al., I96I ; Clausen, I963) ou de maïs (Combs et Wallace, r962 ; Brooks et Thomas, I959; Vipperman et al., I963). C'est essentiellement à la persistance du déficit en lysine qu'on peut attribuer cette infériorité. L'addition de monochlorhydrate de $l$-lysine au tourteau d'arachide permet en effet aux animaux d'exprimer des performances analogues à celles qu'ils pourraient avoir avec la farine de poisson en présence d'orge (EvaNs, I958, I959, I960, I96I ; Jonws et al., I96r, I962) ou le tourteau de soja en présence d'orge (Clausen et al., ig6r, Clausen, i963) ou de maïs (Vipperman et al., ig63) ; tel n'est toutefois pas l'avis de HANDLIN et al. (Ig62) qui se sont placés dans des conditions expérimentales différentes. Il n'est pas possible d'attribuer l'amélioration constatée au simple fait que la consommation est accrue lorsque le régime est additionné de lysine. En effet, dans nos expériences, chez les animaux placés en cage à métabolisme, on constate également cet effet bénéfique de la lysine bien que leur consommation soit ajustée sur celle des témoins. En outre, dans ces conditions, la réiention azotée est améliorée en valeur absolue et en valeur relative, ce qui a été également démontré par JoNEs et al. (I96I). On peut donc conclure que 1'addition de lysine synthétique au régime orge-arachide a rééquilibré les aminoacides entre eux et a, de ce fait, amélioré la valeur biologique de ce mélange protidique.

Il faut cependant noter que la réponse des animaux à une addition de lysine au régime, selon plusieurs doses, n'est pas linéaire lorsque la croissance est libre : si l'optimum de la supplémentation se situe aux environs de 0,2 p. Ioo lors de la première phase de croissance, la dose supérieure provoque une dépression relative de la croissance. Tout se passe done comme s'il existait alors un déséquilibre par excès entraînant des conséquences néfastes pour les animaux. Un tel déséquilibre par excès a déjà été signalé pour la lysine dans un régime maïs-soja (SCHNARRE et TrRIBbLE, Ig62) ou pour les acides aminés soufrés dans un régime complexe (RÉRAT, I,OUGNoN et PION, I962). Aucune explication satisfaisante de ce phénomène ne peut être fournie. Par ailleurs, étant donné que deux taux saulement de supplémentation ont été utilisés dans cette expérience, il n'est pas possible de préciser le besoin en lysine ; tout ce qu'on peut dire est que les meilleures performances des animaux ont été atteintes, dans nos conditions expérimentales (lot $I_{A}$ et lot $I_{C}$ ) grâce à des régimes contenant des taux de lysine compris entre 0,75 et $0,80 \mathrm{p}$. Ioo de la ration pour les animaux de 20 à $60 \mathrm{~kg}$ (soit 4,5 à 5,0 p. Ioo des protéines). Cependant, il faut bien préciser que les performances obtenues sont médiocres et peuvent être dépassées (RÉRAT et HENRY, I964), ce qui serait en faveur de l'apparition d'un facteur limitant autre que la lysine lorsque celle-ci est ajoutée au régime au taux de $2 \mathrm{~g} / \mathrm{kg}$. Pour les animaux dont le poids dépasse $60 \mathrm{~kg}$, un taux de lysine de o,45 p. Ioo est suffisant 
(3,2 p. Ioo des protéines) ; il est du reste connu que le besoin en amino acides indispensables relativement à l'énergie diminue lorsque les animatux prennent de l'âge (Morgan et Robinson, I964) et sur ce point, nos résultats sont proches de ceux d'Evans (I960) et de BRAUDE et al. (I960). Si l'on en juge par nos propres résultats, il semble même que la vitesse de croissance permise par les régimes contenant les protéines les plus déséquilibrées (tourteau d'arachide) soit la plus élevée au cours de la deuxième phase de croissance; il peut toutefois s'agir d'un phénomène de croissance compensatrice puisque c'est précisément chez ces animaux que la croissance a été la plus lente au cours de la première phase de développement (RoBinsos, I964).

Si la présence d'un excès d'un acide aminé indispensable peut se traduire par une dépression de croissance, par contre, l'addition d'un acide aminé non essentiel, comme le glycocolle, en quantité relativement importante ne provoque aucune modification des performances du Porc. Ce résultat est conforme à ceux de HoGAN (I949); d'après RoTH et ALIISON (I949) chez le Rat, il apparaît en outre que le glycocolle peut même avoir une action protectrice vis-à-vis de l'excès d'un acide aminé essentiel et il n'est pas exclu qu'il ait partiellement joué un tel rôle dans nos expériences. A l'encontre de ces conclusions, d'autres auteurs (SAUBERIICH, I96I; SWENDSEID et al., I962) concluent à la possibilité d'un effet dépressif des acides aminés non essentiels chez le Rat; cependant, leurs régimes expérimentaux présentaient des taux azotés très faibles et l'addition de glycocolle réalisait alors un déséquilibre marqué des protéines du régime.

Enfin, sur le plan de la composition corporelle, la variabilité des résultats que nous avons obtenus est extrême. Cependant, l'ađdition de $l$-lysine au régime de base orge-arachide (expérience $\mathrm{C}$ ) se traduit par une augmentation significative du pourcentage des tissus maigres. Il apparaît done que les animaux sont d'autant plus maigres que la valeur biologique des protéines du régime est meilleure; les différences enregistrées au cours de l'expérience A, quoique non significatives, vont dans le même sens. Ces conclusions sont en accord avec celles de Kropf et al. (I959), Jones et al. (I962), Cahilly et al. (I963), Clausen (Ig63), Robinson et Lewis (I963) VIPPERMAN et al. (I963).

En bref, l'addition de quantités modérées de lysine de synthèse à un régime orge-arachide se traduit par une amélioration de la valeur biologique des protéines de ce régime : la vitesse de croissance des animaux est augmentée ainsi que l'efficacité de la rétention azotée; la composition corporelle est améliorée. Les performances des animaux recevant un tel régime sont analogues à celles d'animaux recevant un régime isoazoté orge-poisson. Ces conclusions ne sont valables que pour les animaux relativement jeunes; après qu'ils aient atteint $60 \mathrm{~kg}$ de poids vif, ils ne réagissent pratiquement plus à une modification de la qualité de la source azotée du régime, pour le taux azoté utilisé.

Reçu pour publication en mars 1965

\section{REMERCIEMENTS}

A l'A. E. C., Société de Chimie Organique et Biologique, pour nous avoir foumi gracieusement la $l$-lysine nécessaire à ces essais. 


\section{SUMMARY}

SUPPLEMENTATION OF A SIMILIFIED VEgETABIE DIET FOR GROWING PIGS WITH L-LySINE

In a diet for growing pigs after weaning, fishmeal (norwegian herringmeal) was significantly better than groundnut meal, supplying the same amount of protein, as a supplement to barley. The superiority was shown in growth rate, feed per unit weight gained and efficiency of utilization of protein, both in animals from 20 to $60 \mathrm{~kg}$ and for the whole experiment from 20 to $100 \mathrm{~kg}$. Body composition on the other hand, was not changed when groundnut meal replaced fishmeal.

A supplement of $0.2 \mathrm{p}$. $100 \mathrm{~L}$-lysine hydrochloride to a mixture of barley and groundnut meal in diets for pigs from 20 to $60 \mathrm{~kg}$ gave a significant improvement in the characteristics studied. Performance of the pigs given the supplemented diet was equal to that on a diet of barley and herringmeal. A supplement of 0.4 p. $100 \mathrm{~L}$-lysine IICl to the same diet did not give as good results, but there was no significant difference in performance of the pigs given the different amounts of L-lysine. From $60 \mathrm{~kg}$, a supplement of 0.15 p. 100 lysine (or more) did not give any significant improvement in growth. The carcases of the pigs given the supplements had a significantly higher proportion of lean in ham and loin than in controls. Nitrogen balance estimated in castrated male pigs confirmed the foregoing results : retention of nitrogen increased when the diet of barley and groundnut was supplemented with lysine IHCl.

\section{RÉFÉRENCES BIBIIOGRAPHIQUES}

Becker D. 1.., I958. Proper protein and amino acid levels important in swine feeds. The Feed Bag Red Book. Buyers Guide, 157-164. Editorial Service Company, Milwaukee.

Block R. J., Mrthel. H. H., 1946. The correlation of the amino acid composition of proteins with their nutritive value. Nulr. Abstr. Rev., 16, 249-278.

Braude R., Townsend M. J., Harking'ox G., Rowell J. G., ig6o. Effects of different protein contents in the rations of growing-fattening pigs. J. agric. Sci., 55, 17.5-181.

Brooks C. C., Tiromas II. R., 1959. Supplentents to peanut oil meal protein for growing fattening swine. J. animal Sci., 18, I II9-II27.

Cahilly G. M.jr, Miller R. F., Kelly R. F., Brooks C. C., ig63. Effect of various levels of dietary lysine on certain blood phenomena, muscle development, and muscle-protein biological value of growing swine. J.animal Sci., 22, 726-732.

Clatsen H., Petersen F. H., Højgaard-Olsen N. J., II lansen V., Maiden A., Nielsen H., Eggum B. 0., 1961. Rapport concernant les expériences de reproduction et d'alimentation des porcs réalisées par le laboratoire de recherches sur l'économie rurale el les abattoirs coopératifs danois (en danois). Frederiksberg Bogtrykkeri, Copenhague.

Clausen H., I963. Tas Eiweiss im Futter der Mastschweine. Förderungsdienst, 11, no spécial, 35-42.

Combs G. E., Wallace H. D., 1962. Peanut meal as a source of protein in pig starter and grower rations. J. animal Sci., 21, 95-97.

Dunn M.S., Shankman S., Camien M. N., Frankl W., Rockland L. B., I944. Investigation of amino acids, peptides and proteins. XVIII. The amino acid requirements of Leuconosloc mesenteroides P. 60. J. Biol. Chem., 156, 703-713.

Evaxs R. E., I958. Nutrition of the bacon pig. XIX. The requirement of the bacon pig for certain essential amino acids. J. agric. Sci, $50,230-242$.

Evans R. E., I959. The minimum amount of white fish meal required to supplement the proteins in some vegetable-protein concentrates. J. agric. Sci., 53, $230-246$.

Evans R. E., 1960. The effect of adding lysine and methionine to the diet of pigs kept on low-protein vegetable foods. J.agric. Sci., 54, 266-273.

Evans R. E., 1961. The protein-saving action of synthetic lysine and methionine. J.agric. Sci. 57, I I I121 .

Handlin D. L., Cox J. E., Kropf D. H., Wileeler K. F., ig62. Effect of adding lysine and/or oleandomycin to plant protein rations for swine. J. animal Sci., 21, 378 (Abstr.)

Hogan J. M., I949. Thesis, Rutgers University, cité par Roth J. S., Allison J. B., I950. The effects of feeding excess DL-methionine and choline chloride to rats on a casein diet. J. Biol. Chem. 183, 173-1 78., 
Jones A.S., Hepburn W. R., Boyne A. W., I96I. The nitrogen metabolism of the young pig. I. Suppleneental value of certain amino acids when added to groundnut meal rations. J. Sci. Food Agric., 12, $353^{-3} 3^{62}$.

Jones A. S., Hepburn W. R., Cadenhesid A., Boyne A. W., 1962. The effect of variation of protein quality and protein level in diets on the performances of young pigs. Animal Prod., 4, I85-193.

Kropi: D. H., Bray R. W., Philllips P'. II., Grummer R. II., 1959. Effect of protein level and quality in swine rations upon growth and carcass development. J. animal Sci., 18, 755-7602.

Lison L., 1958 . Statistique appliquée à la binlogie expérintentale. La planification de l'expérience et l'analyse des résultals. Gauthier-Villars, l'aris.

MiTCneLL H. II., I930. The paired feeding method : its value and limitations in livestock experimentation. Rec. Amer. Soc. animal Prod., 23, Ann. Meet., 63-73.

Morgan J. T., Robinson D. W. in Morgan J. T., Lewis D., ig6z. Nutrition of pigs and poultry. Butterworths ed, London, p. 255-285.

Pion R., De Belsunce C., Fauconnead G., i963. Composition en acides aminés de quelques aliments. Ann. Biol. anim. Bioch. Biophys., 3, (n' h. s. I), I I-1 8.

Rérat A., Lougnon J., Pion R., 1962. Supplémentation d'un régime complexe par la DL-méthionine de synthèse, chez le Porc. Ann. Zootech., 11, I 59-172.

Rérat A., Henry Y., ig64. Fitude du besoin azoté chez le Porc en croissance. I. Utilisation de la farine de poisson à trois taux différents. Ann. Zootech., 13, 5-34.

Robisison D. W., Lewis D., 1963. Amino acid supplementation of a barley ration for the pig. J. Sci. Food Agric., 14, 806-813.

Robinson D. W., r 964. The plane of nutrition and compensatory growth in pigs. Anim. Prod., 6, 227-236.

Rotil J. S., Allison J. B., 1949. The effect of feeding excess glycine, L-arginine and DL-methionine to rats on a casein diet. Proc. Soc. Exper. Biol. Med., 70, 327-330.

SACBERLICII II. F., I961. Studies on the toxicity and antagonism of amino acids for weanling rats. J.Nutr., $75,61-7^{2}$.

ScindrRe N. II. A., T'ribble L. F., I962. Lysine nutrition of swine. J. animal Sci, 21, 1013 (Abstr.).

Swenidseid M. E., Hickson J. B., Friedrici B. W., 1962. Iffect of non essential nitrogen supplements on growth and on the amino acid content in plasma and muscle of weanling rats fed a low-protein diet. J. Nutr., 78, II 5-II9.

Vipphrman P. E., jr, Brooks C. C., Kelly R. F., Graham P. P., Thomas H. R., i963. Effect of dietary lysine level on muscle size and composition in swine. J. animal Sci., 22, 674-678. 